\section{Salud mental en población transgénero y género no conforme en Chile}

\author{
MÓNICA GUZMÁN-GONZÁLEZ ${ }^{1, a}$, JAIME BARRIENTOS ${ }^{2, b}$, \\ JOSÉ L. SAIZ ${ }^{3, \mathrm{C}}$, FABIOLA GÓMEZ ${ }^{4, \mathrm{~d}}$, MANUEL CÁRDENAS ${ }^{5, \mathrm{e}}$, \\ RICARDO ESPINOZA-TAPIA ${ }^{1, \mathrm{f}}$, JOAQUÍN BAHAMONDES ${ }^{6, \mathrm{~g}}$, \\ LEONOR LOVERA $^{2, \mathrm{~h}}$, ALAIN GIAMI $^{7, \mathrm{i}}$
}

\section{Mental health in a sample of transgender people}

Background: Transgender population is at a higher risk of suffering mental health problems than cisgender population (people whose sex and gender identity are in agreement) and other sexual minorities (gays, lesbians, and bisexuals), partly due to the greater stress that they suffer or for being victims of sexual prejudice. Aim: To describe positive and negative mental health indicators in a sample of transgender people living in Chile. Material and Methods: An intentional sample of 377 transgender individuals older than 18 years, living in the country's northern, center and southern zones were studied. Sociodemographic and well-being features, negative emotional symptoms, suicidal behavior, and substance consumption were assessed. Results: A precarious mental health was confirmed among participants. A high frequency of depression, ideation and suicide attempts, and high levels of depression, anxiety, and stress symptoms were identified. Also, a high frequency of substance abuse was found. These mental health indicators varied according to the sex assigned at birth and gender self-identification. Conclusions: Transgender people experience precariousness and vulnerability in their mental health.

(Rev Med Chile 2020; 148: 1113-1120)

Key words: Gender Identity; Mental Health; Transgender Persons.
'Escuela de Psicología, Universidad Católica del Norte. Antofagasta, Chile.

${ }^{2}$ Facultad de Psicología, Universidad Alberto Hurtado. Santiago, Chile.

${ }^{3}$ Departamento de Psicología, Universidad de La Frontera. Temuco, Chile.

${ }^{4}$ Escuela de Psicología, Pontificia Universidad

Católica de Chile. Santiago, Chile.

${ }^{5}$ Escuela de Psicología, Universidad de Talca. Talca, Chile.

${ }^{6}$ School of Psychology, University of Auckland. Auckland, Nueva Zelanda.

${ }^{7}$ INSERM. Public Health Institute. París, Francia.

aPsicóloga, Dra. en Psicología.

bPsicóloga, Dr. en Psicología Social.

'Psicólogo, Dr. en Psicología.

'Psicóloga, Magister en Psicología Social,

estudiante de Doctorado en Psicología, Escuela de Psicología, Pontificia Universidad Católica de

Chile, Santiago, Chile.

ePsicólogo, Dr. En Psicología Social.

fPsicólogo, Dr. en Psicología Social.

'Psicólogo, Magister en Psicología Social, estudiante de Doctorado en Psicología,

School of Psychology, University of Auckland. Auckland, Nueva Zelanda.

hSocióloga.

'Psicólogo, Dr. en Psicología Social.

Apoyo financiero: Este artículo fue financiado por la Comisión Nacional de Investigación Científica y Tecnológica (CONICYT) de Chile según el Proyecto FONDECYT 1170046.

La organización que proporcionó el financiamiento no tuvo influencia en el diseño del estudio, la recolección, análisis o interpretación de los datos ni en la preparación revisión o aprobación del manuscrito.

Los autores declaran no tener conflictos de interés

Recibido el 6 de marzo de 2020, aceptado el 18 de agosto de 2020 .

Correspondencia a:

Jaime Barrientos

Facultad de Psicología, Universidad Alberto

Hurtado. Santiago, Chile.

jbarrientos@uahurtado.cl
I

os derechos de las minorías sexuales y de género (MSG) son parte de la agenda pública chilena, especialmente desde la aprobación de la denominada ley antidiscriminación ${ }^{1}$. Pese a ello, en Chile aun persiste un trato inequitativo con estas minorías, particularmente con las personas transgénero ${ }^{2}$.

La palabra transgénero define a personas cuya identidad de género o la expresión de ésta difiere de aquella esperada culturalmente según la asig- nación de sexo masculino o femenino al nacer ${ }^{3,4}$. Diversos estudios ${ }^{5,6,7}$ han señalado que el prejuicio es especialmente elevado, y con efectos más nocivos, cuando está dirigido a personas transgénero en comparación con otras MSG. Ilustrando, las personas transgénero femeninas (aquellas con sexo masculino asignado al nacer que procuran afianzar su género femenino), reportan estar expuestas a alto prejuicio familiar y laboral ${ }^{8}$.

Dado lo anterior, se ha planteado que la 
población transgénero posee mayor riesgo de problemas en salud mental que la población cisgénero (personas cuyo sexo e identidad de género concuerdan) y que otras MSG (gays, lesbianas y bisexuales) debido, en parte, al mayor estrés que sufren por ser víctima de prejuicio sexual ${ }^{9,10}$. Las personas transgénero presentan elevados niveles de depresión, angustia y abuso de sustancias ${ }^{7}$, un riesgo nueve veces mayor de suicidio y alta prevalencia de malestar psicológico grave ${ }^{11,12}$. Además, estas personas tienen menor acceso a cuidados de salud que sean afirmativos de su identidad/ expresión de género ${ }^{13}$.

La investigación sobre la salud mental de las personas transgénero en América Latina es escasa, en contraste con la necesidad de generar conocimiento que fundamente políticas públicas dirigidas a reducir las disparidades de salud existentes en esta población ${ }^{7}$. Igualmente, en Chile, se dispone de pocos estudios sobre esta población, particularmente respecto a su caracterización en términos de salud mental. No obstante, la poca información nacional disponible coincide con la literatura internacional, por ejemplo, respecto a alta prevalencia de suicidio y depresión ${ }^{14,15}$.

Dada la escasez de investigación nacional sobre esta población, el presente estudio se propuso describir la salud mental de una muestra de personas transgénero residentes en Chile. Se hipotetizó que esta muestra presentaría indicadores positivos bajos e indicadores negativos altos.

\section{Materiales y Método}

\section{Participantes}

La muestra, de carácter intencionado y obtenida mediante reclutamiento por "bola de nieve", comprendió 377 participantes que cumplieron con cuatro criterios de inclusión: auto-describirse como transgénero, tener al menos 18 años, residir en Chile y participar voluntariamente en el estudio. Sus edades fluctuaron entre 18 y 67 años $(M=31,88 ; S D=10,48)$. Los participantes residían en Santiago (37,9\%), Valparaíso (34,2\%), Concepción (11,1\%), Antofagasta $(9,0 \%)$ y Arica $(7,7 \%)$. El nivel socioeconómico fue el siguiente: bajo y medio bajo $(51,0 \%)$, medio $(35,1 \%)$, medio alto y alto $(13,9 \%)$. La mayoría $(79,7 \%)$ tenía nacionalidad chilena. El 36,9\% declaró sexo femenino asignado al nacer (SFAN) y $63,1 \%$ sexo masculino asignado al nacer (SMAN).
Un análisis de contenido dirigido ${ }^{16}$ de las respuestas a la pregunta “¿Cómo describirías tu identidad de género en tus propias palabras?" 17,18 permitió clasificar a la muestra en ocho categorías de autoidentificación de género. Suscribiendo la dicotomía convencional de género ${ }^{19}, 13,8 \%$ y $24,1 \%$ se describió como "hombre" o "mujer", respectivamente. El 7,2\% señaló ser "hombre trans" y 22,8\% "mujer trans" aludiendo, así, un sexo junto a una referencia al proceso de afirmación de género. El 8,0\% señaló ser "trans", sin ninguna alusión a la dicotomía de género. El $6,1 \%$ y $3,4 \%$ reportaron una identificación "no binaria" (e.g., "agénero") y "travesti”, respectivamente. Finalmente, en la categoría "otros", 14,6\% no manifestó identificación de género (e.g., "yo mismo") u omitió respuesta.

\section{Medidas}

Se empleó un cuestionario autoadministrado. Se obtuvo información sociodemográfica sobre sexo asignado al nacer, edad, ciudad de residencia, nacionalidad, nivel educacional, y trabajo actual. El procedimiento ESOMAR ${ }^{20}$ fue empleado para determinar el nivel socioeconómico.

Como indicador positivo de salud mental se evaluó la satisfacción con la vida mediante la escala homónima, en la versión validada para Chile ${ }^{21}$. Es una escala unidimensional, conformada por cinco ítems con seis opciones de respuesta desde 1 (completamente en desacuerdo) hasta 6 (completamente de acuerdo). Su coeficiente de fiabilidad $\alpha$ en este estudio fue 0,84 .

Se empleó también la versión validada para Chile $^{22}$ del instrumento Abbreviated Scales of Depression, Anxiety and Stress (DASS-21) ${ }^{23}$. Esta escala contiene 21 ítems que miden sintomatología depresiva, ansiosa y estrés durante la última semana. Las opciones de respuesta van desde 0 (no describe nada de lo que me pasó o sentí durante la semana) hasta 3 (sí, esto me pasó mucho o casi siempre). En este estudio, la confiabilidad $\alpha$ fue $0,87,0,84$ y 0,87 para las subescalas depresión, ansiedad y estrés, respectivamente. Además, se incluyeron dos preguntas ${ }^{24}$ de respuesta si/no sobre depresión: "¿Alguna vez un médico te ha diagnosticado depresión?” y “iAlguna vez has recibido tratamiento por depresión?”.

La Escala de Suicidalidad de Okasha ${ }^{25}$ validada en Chile $^{26}$ fue administrada para indagar riesgo suicida. Está conformada por tres ítems 
que abordan ideación suicida y uno que registra intentos suicidas. Se usaron seis opciones de respuesta desde 1 (nunca) hasta 6 (muchas veces) y, además, se instruyó responder considerando los últimos 12 meses. Los puntajes de ideación suicida $(\alpha=0,89)$ y de intentos suicidas fueron analizados por separado.

Se aplicó también la versión autoadministrada adaptada para Chile ${ }^{27}$ del Alcohol, Smoking and Substance Involvement Screening Test (ASSIST) ${ }^{28}$. Esta versión evalúa la frecuencia de consumo de siete sustancias según cinco opciones de respuesta desde 0 (no has consumido en los últimos tres meses) hasta 4 (consumo diario, 5 a 7 veces por semana). Las sustancias fueron: tabaco, alcohol, marihuana, cocaína, inhalantes, anfetaminas u otros estimulantes, y pasta base de cocaína. Para comparar con resultados en población general, se consideró como prevalencia un consumo mensual ( 1 a 3 veces en el último mes) o mayor.

\section{Procedimiento}

Habiendo conocido los objetivos del estudio, los participantes firmaron un consentimiento informado que garantizaba una participación voluntaria y anónima y la confidencialidad de los datos. Este estudio fue aprobado por el Comité de Ética de la Universidad de Santiago de Chile.

\section{Análisis de datos}

Se realizaron análisis descriptivos en la muestra total e inferenciales $\left(\chi^{2}, t\right.$, ANOVA y tamaño del efecto) comparando cada indicador de salud mental según sexo asignado al nacer y autoidentificación de género.

\section{Resultados}

\section{Salud mental en la muestra total}

El $40,0 \%$ de todos los participantes ha sido alguna vez diagnosticado por depresión y $33,3 \%$ ha recibido tratamiento por ello. El $68,8 \%$ ha tenido ideación suicida en el último año y, entre ellos, $51,5 \%$ ha pensado en suicidarse. Además, $34,1 \%$ reporta al menos un intento suicida en el último año. El 10,1\% señala haber consumido tabaco, 25,5\% alcohol, 10,3\% marihuana, 6,4\% cocaína, $1,6 \%$ anfetaminas $u$ otro estimulante y $1,6 \%$ pasta base de cocaína, con una frecuencia igual o mayor a 1-3 veces mensuales.

La Tabla 1 presenta los estadísticos descriptivos de salud mental en la muestra total. Aunque el

Tabla 1. Indicadores de salud mental según muestra total y sexo asignado al nacer

\begin{tabular}{|c|c|c|c|c|}
\hline Indicadores & Rango & $\begin{array}{c}\text { Total } \\
(\mathbf{n}=\mathbf{3 7 7}) \\
M \text { (DE) }\end{array}$ & $\begin{array}{c}\text { SMAN } \\
(n=238) \\
M \text { (DE) }\end{array}$ & $\begin{array}{c}\text { SFAN } \\
(n=139) \\
M(D E)\end{array}$ \\
\hline Satisfacción con la vida & $1-6$ & $4,27(1,30)$ & $4,47(1,28)$ & $3,91(1,27)$ \\
\hline Síntomas de depresión & $0-21$ & $6,30(0,75)$ & $5,95(0,73)$ & $6,93(0,78)$ \\
\hline Síntomas de ansiedad & $0-21$ & $6,51(0,75)$ & $6,44(0,78)$ & $6,65(0,70)$ \\
\hline Síntomas de estrés & $0-21$ & $7,63(0,78)$ & $7,00(0,78)$ & $8,68(0,77)$ \\
\hline Ideación suicida & $1-6$ & $2,60(1,67)$ & $2,22(1,49)$ & $3,24(1,76)$ \\
\hline Intentos de suicidio & $1-6$ & $1,86(1,52)$ & $1,82(1,53)$ & $1,94(1,51)$ \\
\hline \multicolumn{5}{|l|}{ Consumo de } \\
\hline Tabaco & $0-4$ & $1,74(1,69)$ & $1,70(1,70)$ & $1,81(1,68)$ \\
\hline Alcohol & $0-4$ & $1,74(1,34)$ & $1,70(1,38)$ & $1,82(1,26)$ \\
\hline Marihuana & $0-4$ & $1,49(1,66)$ & $1,48(1,71)$ & $1,51(1,57)$ \\
\hline Cocaína & $0-4$ & $0,60(1,15)$ & $0,72(1,23)$ & $0,39(0,96)$ \\
\hline Inhalantes & $0-4$ & $0,17(0,66)$ & $0,22(0,74)$ & $0,10(0,49)$ \\
\hline Anfetaminas & $0-4$ & $0,19(0,72)$ & $0,19(0,74)$ & $0,18(0,69)$ \\
\hline Pasta base & $0-4$ & $0,36(1,01)$ & $0,49(1,16)$ & $0,12(0,52)$ \\
\hline
\end{tabular}

Nota. $M=$ media; $D E$ = desviación estándar; SMAN = sexo masculino asignado al nacer: SFAN = sexo femenino asignado al nacer. 
promedio de satisfacción con la vida es moderadamente alto, los promedios de síntomas de depresión, ansiedad y estrés son superiores a los puntos de corte ( $>5,>4 \mathrm{y}>5$, respectivamente) establecidos para jóvenes chilenos ${ }^{29}$ indicando una presencia relevante de síntomas emocionales negativos.

Como se anticipó, los resultados en la muestra total señalan que las personas transgénero poseen una precaria salud mental, al menos en cuanto a depresión y otros síntomas emocionales negativos, ideación e intentos suicidas y consumo de sustancias.

\section{Salud mental según sexo asignado al nacer}

Poseer o no diagnóstico de depresión se asocia significativamente con el sexo asignado al nacer, evidenciando las personas con SFAN mayor prevalencia de depresión $(58,3 \%)$ que las personas con $\operatorname{SMAN}(30,5 \%), \chi^{2}(1)=27,92, \mathrm{p}<0,001, V=0,27$. Asimismo, una mayor proporción de personas con SFAN $(51,1 \%)$ que con SMAN $(22,9 \%)$ recibió tratamiento por depresión, $\chi^{2}(1)=31,30$, $\mathrm{p}<0,001, \mathrm{~V}=0,29$.

En la Tabla 1, cinco indicadores difieren significativamente en función del sexo asignado al nacer. Aunque los participantes con SMAN poseen mayor satisfacción con la vida, $\mathrm{t}(375)=$ $4,13, \mathrm{p}<0,001, \mathrm{~d}=0,44$, reportan mayor consumo de cocaína, $\mathrm{t}(373)=2,73, \mathrm{p}=0,007, \mathrm{~d}=0,30$ y de pasta base, $\mathrm{t}(267)=2,83, \mathrm{p}=0,005, \mathrm{~d}=0,41$. Por su parte, las personas con SFAN presentan mayor presencia de síntomas de estrés, $\mathrm{t}(375)=2,83$, $\mathrm{p}=0,005, \mathrm{~d}=0,31, \mathrm{y}$ mayor frecuencia de ideación suicida en el último año, $\mathrm{t}(373)=5,97, \mathrm{p}<0,001$, $\mathrm{d}=0,63$.

\section{Salud mental según autoidentificación de género}

Poseer o no diagnóstico de depresión se asocia significativamente con autoidentificación de género, $\chi^{2}(7)=29,88, p<0,001, V=0,28$. Específicamente, tres grupos exhiben frecuencias de diagnóstico mayores al 50\%: "no binario" $(69,6 \%)$, "hombre" (59,6\%) y "mujer trans" (51,9\%). Igualmente, el haber o no recibido tratamiento por depresión se vincula significativamente con autoidentificación de género, $\chi^{2}(7)=35,30, p<0,001$, $\mathrm{V}=0,31$. Solo el grupo "no binario" posee una frecuencia de tratamiento mayor a 50\% $(65,2 \%)$.

La Tabla 2 presenta los estadísticos descriptivos de los demás indicadores de salud mental según autoidentificación de género. Solo se constatan relaciones significativas entre autoidentificación de género y los niveles de satisfacción con la vida, $\mathrm{F}(7,369)=4,59, \mathrm{p}<0,001, \mathrm{~d}=0,31$, ideación suicida, $\mathrm{F}(7,367)=4,83, p<0,001, d$ $=0,29$, y consumo de inhalantes, $\mathrm{F}(7,351)=$ $3,26, \mathrm{p}<0,01, \mathrm{~d}=0,25$, y pasta base, $\mathrm{F}(7,261)=$ $2,63, p=0,01 ; d=0,31$. Análisis post hoc revelan que quienes se autodefinen como "mujer trans" reportan niveles significativamente más altos de satisfacción con la vida que quienes se autodefinen como "hombre", "hombre trans" y "no binario". Por su parte, las personas que se autodefinen como "trans" reportan niveles de satisfacción mayores en contraste con quienes lo hacen como "no binario". Finalmente, aquellas personas en la categoría "otro" presentan niveles de satisfacción mayores que aquellos que se autodefinen como "mujer trans". En relación a ideación suicida, los dos grupos con promedios superiores, "no binario" y "hombre trans", reportan pensamiento suicida significativamente más frecuente que los tres grupos con promedios inferiores, "mujer trans", "mujer" y "trans". En la asociación de identificación de género con consumo de inhalantes y pasta base, los análisis post hoc no revelan diferencias significativas.

\section{Discusión}

Este estudio describió indicadores de salud mental en personas transgénero que viven en Chile. Tal descripción fue efectuada en la muestra total y, además, en función del sexo asignado al nacer y la autodefinición de género.

El nivel moderadamente alto de satisfacción con la vida observado en la muestra total es similar al registrado en otras MSG chilenas ${ }^{30}$ y coincidente con evidencia que describe a los chilenos, $y$ latinoamericanos, como personas subjetivamente felices, incluso en contextos desfavorables ${ }^{31}$.

Los resultados obtenidos en los indicadores negativos de salud mental refrendan la literatura respecto a una marcada presencia de problemas de salud mental en esta población. Con la cautela que impone el análisis de una muestra no probabilística, los participantes transgénero del presente estudio reporta una prevalencia de depresión $(40,0 \%)$ diez veces mayor que la población general a nivel mundial $(4,4 \%)^{32}$ y casi siete veces 
Tabla 2. Indicadores de salud mental según autoidentificación de género

\begin{tabular}{|c|c|c|c|c|c|c|c|c|}
\hline Indicadores & $\begin{array}{l}\text { Hombre } \\
(n=52)\end{array}$ & $\begin{array}{l}\text { Mujer } \\
\text { (n= 91) }\end{array}$ & $\begin{array}{c}\text { Hombre } \\
\text { trans } \\
(n=27)\end{array}$ & $\begin{array}{c}\text { Mujer } \\
\text { trans } \\
(n=86)\end{array}$ & $\begin{array}{c}\text { Trans } \\
(n=30)\end{array}$ & $\begin{array}{c}\text { No } \\
\text { binario } \\
(n=23)\end{array}$ & $\begin{array}{l}\text { Travesti } \\
(n=13)\end{array}$ & $\begin{array}{c}\text { Otro } \\
(n=55)\end{array}$ \\
\hline Satisfacción con la vida & $\begin{array}{r}3,97^{a} \\
(1,25)\end{array}$ & $\begin{array}{c}4,35 \\
(1,34)\end{array}$ & $\begin{array}{c}3,79^{b} \\
(1,39)\end{array}$ & $\begin{array}{c}4,70^{\text {abcd }} \\
(1,07)\end{array}$ & $\begin{array}{l}4,78^{e} \\
(1,18)\end{array}$ & $\begin{array}{l}3,58^{\text {ce }} \\
(1,28)\end{array}$ & $\begin{array}{c}4,29 \\
(1,16)\end{array}$ & $\begin{array}{l}4,96^{d} \\
(1,40)\end{array}$ \\
\hline Síntomas de depresión & $\begin{array}{c}5,25 \\
(0,62)\end{array}$ & $\begin{array}{c}6,09 \\
(0,83)\end{array}$ & $\begin{array}{c}8,54 \\
(0,87)\end{array}$ & $\begin{array}{c}6,02 \\
(0,68)\end{array}$ & $\begin{array}{c}6,09 \\
(0,75)\end{array}$ & $\begin{array}{c}6,86 \\
(0,83)\end{array}$ & $\begin{array}{c}6,44 \\
(0,65)\end{array}$ & $\begin{array}{c}6,86 \\
(0,77)\end{array}$ \\
\hline Síntomas de ansiedad & $\begin{array}{c}6,02 \\
(0,78)\end{array}$ & $\begin{array}{c}6,09 \\
(0,67)\end{array}$ & $\begin{array}{c}6,59 \\
(0,77)\end{array}$ & $\begin{array}{c}6,86 \\
(0,80)\end{array}$ & $\begin{array}{c}6,37 \\
(0,86)\end{array}$ & $\begin{array}{c}6,30 \\
(0,75)\end{array}$ & $\begin{array}{c}5,67 \\
(0,58)\end{array}$ & $\begin{array}{c}7,49 \\
(0,73)\end{array}$ \\
\hline Síntomas de estrés & $\begin{array}{c}7,77 \\
(0,81)\end{array}$ & $\begin{array}{c}6,72 \\
(0,79)\end{array}$ & $\begin{array}{c}9,24 \\
(0,81)\end{array}$ & $\begin{array}{c}7,56 \\
(0,77)\end{array}$ & $\begin{array}{c}7,21 \\
(0,90)\end{array}$ & $\begin{array}{c}8,47 \\
(0,75)\end{array}$ & $\begin{array}{c}6,51 \\
(0,63)\end{array}$ & $\begin{array}{c}8,33 \\
(0,71)\end{array}$ \\
\hline Ideación suicida & $\begin{array}{c}2,48 \\
(1,79)\end{array}$ & $\begin{array}{l}2,24^{\text {be }} \\
(1,57)\end{array}$ & $\begin{array}{l}3,47^{\text {def }} \\
(1,87)\end{array}$ & $\begin{array}{l}2,26^{c f} \\
(1,50)\end{array}$ & $\begin{array}{l}2,02^{\text {ad }} \\
(1,34)\end{array}$ & $\begin{array}{c}3,6^{\mathrm{abc}} \\
(1,82)\end{array}$ & $\begin{array}{c}3,41 \\
(1,24)\end{array}$ & $\begin{array}{c}2,77 \\
(1,65)\end{array}$ \\
\hline Intentos de suicidio & $\begin{array}{c}2,12 \\
(1,68)\end{array}$ & $\begin{array}{c}1,65 \\
(1,35)\end{array}$ & $\begin{array}{c}1,74 \\
(1,06)\end{array}$ & $\begin{array}{c}2,08 \\
(1,75)\end{array}$ & $\begin{array}{c}1,50 \\
(1,11)\end{array}$ & $\begin{array}{c}1,57 \\
(1,24)\end{array}$ & $\begin{array}{c}2,62 \\
(2,36)\end{array}$ & $\begin{array}{c}1,85 \\
(1,46)\end{array}$ \\
\hline \multicolumn{9}{|l|}{ Consumo de: } \\
\hline Tabaco & $\begin{array}{c}1,80 \\
(1,81)\end{array}$ & $\begin{array}{c}1,91 \\
(1,68)\end{array}$ & $\begin{array}{c}1,63 \\
(1,64)\end{array}$ & $\begin{array}{c}1,56 \\
(1,61)\end{array}$ & $\begin{array}{c}2,32 \\
(1,81)\end{array}$ & $\begin{array}{c}1,13 \\
(1,39)\end{array}$ & $\begin{array}{c}2,00 \\
(1,96)\end{array}$ & $\begin{array}{c}1,95 \\
(1,67)\end{array}$ \\
\hline Alcohol & $\begin{array}{c}1,65 \\
(1,27)\end{array}$ & $\begin{array}{c}1,60 \\
(1,34)\end{array}$ & $\begin{array}{c}1,52 \\
(1,25)\end{array}$ & $\begin{array}{c}1,69 \\
(1,33)\end{array}$ & $\begin{array}{c}2,25 \\
(1,53)\end{array}$ & $\begin{array}{c}2,04 \\
(1,33)\end{array}$ & $\begin{array}{c}1,85 \\
(1,46)\end{array}$ & $\begin{array}{c}1,85 \\
(1,31)\end{array}$ \\
\hline Marihuana & $\begin{array}{c}1,32 \\
(1,56)\end{array}$ & $\begin{array}{c}1,24 \\
(1,71)\end{array}$ & $\begin{array}{c}1,48 \\
(1,50)\end{array}$ & $\begin{array}{c}1,42 \\
(1,63)\end{array}$ & $\begin{array}{c}1,93 \\
(1,75)\end{array}$ & $\begin{array}{c}2,00 \\
(1,62)\end{array}$ & $\begin{array}{c}2,08 \\
(1,89)\end{array}$ & $\begin{array}{c}1,57 \\
(1,66)\end{array}$ \\
\hline Cocaína & $\begin{array}{c}0,33 \\
(0,88)\end{array}$ & $\begin{array}{c}0,51 \\
(0,93)\end{array}$ & $\begin{array}{c}0,56 \\
(1,09)\end{array}$ & $\begin{array}{c}0,78 \\
(1,32)\end{array}$ & $\begin{array}{c}0,96 \\
(1,58)\end{array}$ & $\begin{array}{c}0,26 \\
(0,86)\end{array}$ & $\begin{array}{c}0,77 \\
(1,24)\end{array}$ & $\begin{array}{c}0,65 \\
(1,21)\end{array}$ \\
\hline Inhalantes & -- & $\begin{array}{c}0,13 \\
(0,52)\end{array}$ & $\begin{array}{c}0,04 \\
(0,19)\end{array}$ & $\begin{array}{c}0,24 \\
(0,76)\end{array}$ & $\begin{array}{c}0,67 \\
(1,33)\end{array}$ & $\begin{array}{c}0,17 \\
(0,84)\end{array}$ & -- & $\begin{array}{c}0,15 \\
(0,54)\end{array}$ \\
\hline Anfetaminas & $\begin{array}{c}0,16 \\
(0,72)\end{array}$ & $\begin{array}{c}0,07 \\
(0,45)\end{array}$ & $\begin{array}{c}0,15 \\
(0,47)\end{array}$ & $\begin{array}{c}0,20 \\
(0,74)\end{array}$ & $\begin{array}{c}0,59 \\
(1,34)\end{array}$ & $\begin{array}{c}0,17 \\
(0,83)\end{array}$ & $\begin{array}{c}0,31 \\
(0,86)\end{array}$ & $\begin{array}{c}0,17 \\
(0,62)\end{array}$ \\
\hline Pasta base & $\begin{array}{c}0,04 \\
(0,19)\end{array}$ & $\begin{array}{c}0,48 \\
(1,15)\end{array}$ & $\begin{array}{c}0,04 \\
(0,21)\end{array}$ & $\begin{array}{c}0,36 \\
(1,04)\end{array}$ & $\begin{array}{c}1,00 \\
(1,68)\end{array}$ & $\begin{array}{c}0,10 \\
(0,44)\end{array}$ & $\begin{array}{c}0,58 \\
(1,00)\end{array}$ & $\begin{array}{c}0,27 \\
(0,79)\end{array}$ \\
\hline
\end{tabular}

Nota. Promedios en una fila con el mismo superíndice difieren significativamente con $\mathrm{p}<0,05$ según la prueba HSD de Tukey. Las personas que se autoidentifican como "hombre" y "travesti" no reportaron consumo de inhalantes.

mayor que la población chilena ${ }^{33}$. Igualmente, el porcentaje de participantes que declara ideación suicida en el último año $(68,8 \%)$ supera con creces al 2,2\% a nivel nacional; lo mismo sucede con los intentos suicidas $(34,1 \% \text { versus } 0,7 \%)^{33}$. Esta mayor prevalencia es también congruente con hallazgos previos en población transgénero en Chile $^{15}$. Por otra parte, la prevalencia de síntomas de depresión, ansiedad y estrés entre los participantes es bastante superior a la reportada por jóvenes chilenos ${ }^{29}$, un sector con predominio de síntomas emocionales negativos. Finalmente, en comparación con la población general ${ }^{34}$, aunque el consumo de alcohol y tabaco entre las personas transgénero es menor, se observa un consumo mayor de marihuana, cocaína y pasta base.
Estos resultados confirman la existencia de una salud mental deficitaria en esta población. Esta situación podría ser consecuencia del rechazo social que históricamente padecen las personas transgénero en Chile ${ }^{6,15}$. Las MSG enfrentan estresores hostiles únicos vinculados con su identidad sexual y/o de género que, al ser crónicos, deterioran su salud $^{14,35}$.

Los problemas de salud mental no se distribuyen homogéneamente en la muestra estudiada. Si bien las personas con SMAN están más satisfechas con su vida, reportan mayor consumo de cocaína y pasta base que las personas con SFAN. Por otra parte, estas últimas presentan mayor frecuencia de diagnóstico y tratamiento de depresión, más síntomas de estrés y mayor 
frecuencia de ideación suicida, que las personas con SMAN. Estos resultados difieren, en parte, del mayor riesgo en salud mental detectado previamente en personas con SMAN en Chile ${ }^{8}$. Al parecer, el proceso de afirmación de género de las personas con SFAN tendría complejidades propias hasta ahora no suficientemente advertidas.

La autoidentificación de género parece ser también un aspecto relevante en la salud mental de las personas transgénero y pone en evidencia dimensiones que no aparecen tan bien cuando solo se usa el sexo asignado al nacer. Quienes se autoidentifican como "no binario" poseen los niveles más bajos de satisfacción vital, siendo inferiores a quienes que lo hacen como "mujer trans" y "trans". Esto podría indicar que las personas que no adhieren a las categorías binarias hombre/mujer tienen experiencias vitales distintas a quienes se adscriben a alguna categoría fundada en el binarismo de género ${ }^{2}$.

Otro hallazgo relevante es la mayor prevalencia de ideación suicida entre quienes se autoidentifican como "no binario" versus quienes lo hacen como "mujer trans", "mujer" o "trans". La categoría "no binario" posee también alta frecuencia de diagnóstico y tratamiento de depresión. Aunque estudios en otros contextos reportan que las personas no binarias, en comparación con aquellas de categorías binarias, presentan peores indicadores de salud ${ }^{36,37}$, otros estudios evidencian menos tentativas suicidas y búsqueda de ayuda por depresión o ansiedad ${ }^{38}$. Con todo, los resultados del presente estudio sugieren que, en Chile, el grupo "no binario" presenta características específicas que deben ser consideradas tanto en la investigación como en el abordaje profesional de la salud en la población transgénero. Parecería que los estresores vinculados al rechazo social hacia las $\mathrm{MSG}^{14,35}$ afectan en mayor medida a las personas transgénero que resisten una imposición normativa binaria; las personas no binarias, al tiempo que reivindican una posición no normativa, deben lidiar con la exclusión social que tal decisión genera ${ }^{39}$.

Debido a dificultades de acceso a la población estudiada fue imposible contar con una muestra probabilística y, por tanto, la generalización de estos hallazgos debe hacerse con cautela. Con todo, el tamaño de la muestra es bastante mayor al empleado en otros estudios nacionales.

\section{Conclusiones}

Las personas transgénero examinadas presentan una deteriorada salud mental. Esta situación, sin embargo, no se distribuye homogéneamente: existen diferencias relevantes según el sexo asignado al nacer y la autoidentificación de género. Para un cometido cabal respecto a las personas transgénero, resulta ineludible que las políticas y planes de salud ${ }^{40}$ y la atención médica ${ }^{7}$ consideren tanto esta deficitaria salud mental como sus variaciones intra-poblacionales.

Agradecimientos: Agradecemos al Institut National de la Santé et de la Recherche Médicale y la Agence Nationale de Recherche sur le SIDA et les Hépatites, ambos de Francia, y The Williams Institute, de la University of California at Los Angeles, EE.UU., su colaboración académica en el desarrollo del proyecto que generó este artículo. Agradecemos también a las organizaciones sociales Organizando Trans Diversidades, Corporación Chilena de Personas Trans Amanda Jofré y a Patricia Casanova por su colaboración en el reclutamiento de participantes y la revisión de la pertinencia del cuestionario empleado y de la interpretación de los resultados.

\section{Referencias}

1. Ley No 20.609. Establece medidas contra la discriminación [Internet]. Santiago, Chile: Ministerio Secretaría General de Gobierno; 24 de julio de 2012. Disponible en: http://bcn.cl/1uyqt.

2. Barrientos, J. Violencia homofóbica en América Latina y Chile. Santiago, Chile: El Buen Aire S.A.; 2015. 154 p.

3. Davidson M. Seeking refuge under the umbrella: Inclusion, exclusion, and organizing within the category Transgender. Sex Res Soc Policy 2007; 4 (4): 60-80. http://dx.doi.org/10.1525/srsp.2007.4.4.60.

4. Valentine D. Imagining transgender: An ethnography of a category. Durham, NC: Duke University Press; 2007. http://dx.doi.org/10.1215/9780822390213.

5. Barrientos J, Cárdenas M. Construction and validation of a subjective scale of stigma and discrimination (SISD) for the gay men and transgender women population in Chile. Sex Res Soc Policy 2014; 11 (3): 187-98. https:// doi.org/10.1007/s13178-014-0150-0.

6. Barrientos J. Situación social y legal de gays, lesbianas y personas transgénero y la discriminación contra 
estas poblaciones en América Latina. Sex Sal y Soc 2016; 22: 331-54. http://dx.doi.org/10.1590/1984-6487. sess.2016.22.15.a.

7. Organización Panamericana de la Salud. Por la salud de las personas trans: Elementos para el desarrollo de la atención integral de personas trans y sus comunidades en Latinoamérica y el Caribe [Internet]. Buenos Aires, Argentina: OPS; 2016. 182 p. Disponible en: http://www. paho.org/arg/images/gallery/Blueprint $\% 20$ Trans $\% 20$ Espa \pm \pm ol.pdf

8. Barrientos J, Gómez F, Cárdenas M. Subjetive well-being and levels of clinical symptomatology in a transwomen sample and men who have sex with men in Chile. J Homosex 2016; 63 (11): 1502-16. https://doi.org/10.1080/ 00918369.2016 .1223346 .

9. Bockting W, Coleman E, Deutsch M, Guillamon A, Meyer I, Meyer W, et al. Adult development and quality of life of transgender and gender nonconforming people. Curr Opin Endocrinol Diabetes Obes 2016; 23 (2): 18897. https://doi.org/10.1097/MED.0000000000000232.

10. Dhejne C, Van Vlerken R, Heylens G, Arcelus J. Mental health and gender dysphoria: A review of the literature. Int Rev Psychiatry 2016; 28 (1): 44-57. https://doi.org/1 $0.3109 / 09540261.2015 .1115753$.

11. Tomicic A, Gálvez C, Quiroz C, Martínez C, Fontbona J, Rodríguez J, et al. Suicidio en poblaciones lesbiana, gay, bisexual y trans: Revisión sistemática de una década de investigación (2004-2014). Rev Med Chile 2016; 144 (6): 723-33. https://doi.org/10.4067/S003498872016000600006.

12. James SE, Herman JL, Rankin S, Keisling M, Mottet L, Anafi M. The report of the 2015 U.S. Transgender Survey [Internet]. Washington, DC: National Center for Transgender Equality; 2016. 297 p. Available from: www.USTransSurvey.org.

13. Fredriksen-Goldsen KI, Simoni JM, Kim H-J, Lehavot K, Walters KL, Yang J, et al. The health equity promotion model: Reconceptualization of lesbian, gay, bisexual, and transgender (LGBT) health disparities. Am J Orthopsychiatry 2014; 84 (6): 653-63. http://dx.doi. org/10.1037/ort0000030.

14. Barrientos J, Espinoza-Tapia R, Meza P, Saiz JL, Cárdenas $\mathrm{M}$, Guzmán-González M, et al. Efectos del prejuicio sexual en la salud mental de personas transgénero chilenas desde el Modelo de Estrés de las Minorías: Una aproximación cualitativa. Terapia Psicológica 2019; 37 (3): 181-97. https://doi.org/10.4067/s071848082019000300181 .

15. Organizando Trans Diversidades. (2017). Informe sobre Encuesta T: $1^{\text {era }}$ Encuesta para personas trans y de género no-conforme en Chile. Resumen ejecutivo [Internet].
Santiago, Chile: OTD; 2017. 37 p. Disponible en: http:// decs.pjud.cl/documentos/descargas/Informe_ejecutivo_Encuesta-T.pdf.

16. Hsieh HF, Shannon SE. Three approaches to qualitative content analysis. Qual Health Res 2005; 15 (9): 1277-88. https://doi.org/10.1177/1049732305276687.

17. Giami A, Beaubatie E. Gender identification and sex reassignment surgery in the trans population: A survey study in France. Arch Sex Behav 2014; 43 (8): 1491-501. http://dx.doi.org/10.1007/s10508-014-0382-3.

18. Carrara S, Hernandez JG, Uziel AP, Conceição GMS, Panjo H, Baldanzi AC et al. Body construction and health itineraries: A survey among travestis and trans people in Rio de Janeiro, Brazil. Cad Saúde Pública 2019; 35 (4): 1-14. http://dx.doi.org/10.1590/0102-311xer110618.

19. Bockting WO. Psychotherapy and the real-life experience: From gender dichotomy to gender diversity. Sexologies 2008; 17 (4): 211-24. https://doi.org/10.1016/j. sexol.2008.08.001.

20. ADIMARK. El nivel socioeconómico ESOMAR. Manual de aplicación [Internet]. Santiago, Chile: ADIMARK; 2000. 8 p. Disponible en: https://www.microweb.cl/idm/ documentos/ESOMAR.pdf.

21. Cárdenas M, Barrientos J, Bilbao M, Páez D, Gómez F, Asún D. Estructura factorial de la Escala de Satisfacción con la Vida en una muestra de estudiantes universitarios chilenos. Rev Mex Psicol 2012; 29 (2): 157-64. https:// www.redalyc.org/articulo.oa?id=243030190006.

22. Antúnez Z, Vinet EV. Escalas de Depresión, Ansiedad y Estrés (DASS-21): Validación de la versión abreviada en estudiantes universitarios chilenos. Terapia Psicológica 2012; 30 (3): 49-55. http://dx.doi.org/10.4067/s071848082012000300005.

23. Lovibond PF, Lovibond SH. The structure of negative emotional states: Comparison of the Depression Anxiety Stress Scales (DASS) with the Beck Depression and Anxiety Inventories. Behav Res Ther 1995; 33 (3): 335-43. http://dx.doi.org/ 10.1016/00057967(94)00075-U.

24. Ministerio de Salud. Presentación F-1. Cuestionario III Encuesta Nacional de Salud 2016-2017 [Internet]. Santiago, Chile: MINSAL; 2016. 59 p. Disponible en: http:// epi.minsal.cl/wp-content/uploads/2018/05/ENS_F1_corr8Mayo.pdf

25. Okasha A, Lotaif F, Sadek A. Prevalence of suicidal feelings in a sample of non-consulting medical students. Acta Psychiatr Scand 1981; 63 (5): 409-15. https://doi. org/10.1111/j.1600-0447.1981.tb00690.x.

26. Salvo GL, Melipillán AR, Castro SA. Confiabilidad, validez y punto de corte para escala de screening de suicidalidad en adolescentes. Rev Chil Neuro-Psiquiatr 
2009; 47 (1): 16-23. http://dx.doi.org/10.4067/S071792272009000100003.

27. Vinet EV, Boero P, Labbé C, Saiz JL. Informe final. Proyecto FONDECYT 1150095: Adultez emergente y variables socioculturales y de salud mental en universitarios chilenos. Temuco, Chile: Universidad de La Frontera, Departamento de Psicología; 2018.

28. Organización Mundial de la Salud. La prueba de detección de consumo de alcohol, tabaco y sustancias (ASSIST). Manual para uso en la atención primaria [Internet]. Washington D.C.: OMS; 2011. 68 p. Disponible en: https://www.who.int/substance_abuse/activities/ assist_screening_spanish.pdf?ua=1.

29. Román F, Santibáñez P, Vinet EV. Uso de las Escalas de Depresión Ansiedad y Estrés (DASS-21) como instrumento de tamizaje en jóvenes con problemas clínicos. Acta de Investigación Psicológica 2016; 6 (1): 2325-36. https://doi.org/10.1016/S2007-4719(16)30053-9.

30. Barrientos J, Gómez F, Cárdenas M, Guzmán M, Bahamondes J. Medidas de salud mental y bienestar subjetivo en una muestra de hombres gays y mujeres lesbianas en Chile. Rev Med Chile 2017; 145 (9): 1115-21. http:// dx.doi.org/10.4067/s0034-98872017000901115.

31. Rojas M. Happiness in Latin America has social foundations. In: Helliwell J, Layard R, Sachs J, editors. World Happiness Report 2018 [Internet]. New York: Sustainable Development Solutions Network; 2018. Chapter 6. Available from: https://s3.amazonaws.com/ happiness-report/2018/WHR_web.pdf.

32. World Health Organization. Depression and other common mental disorders: Global health estimates [Internet]. Geneva: World Health Organization 2017. 21 p. Available from: http://apps.who.int/iris/bitstream/10665/254610/1/WHO-MSDMER- 2017.2-eng. pdf.

33. Ministerio de Salud. Encuesta Nacional de Salud 20162017. Segunda entrega de resultados [Internet]. Santiago, Chile: Departamento de Epidemiología División de Planificación Sanitaria, Subsecretaría de Salud Pública; 2018. 58 p. Disponible en: https://www.minsal.cl/
wp-content/uploads/2018/01/2-Resultados-ENS_MINSAL_31_01_2018.pdf.

34. Observatorio Chileno de Drogas. (2017) Décimo segundo estudio nacional de drogas en población general de Chile, 2016[Internet]. Santiago, Chile: Servicio Nacional para la Prevención y Rehabilitación del Consumo de Drogas y Alcohol; 2017. 301 p. Disponible en: http:// www.senda.gob.cl/wp-content/uploads/media/estudios/ PG/2016_Estudio_Drogas_Poblacion_General.pdf.

35. Meyer I. Prejudice, social stress, and mental health in lesbian, gay, and bisexual populations: Conceptual issues and research evidence. Psychol Bull 2003; 129 (5): 674-97. https://doi.org/10.1037/0033-2909.129.5.674.

36. Burgwal A, Gvianishvili N, Hård V, Kata J, García Nieto I, Orre C, et al. Health disparities between binary and non binary trans people: A community-driven survey. Int J Transgend 2019; 20 (2-3): 218-29. http://dx.doi.or g/10.1080/15532739.2019.1629370.

37. Thorne N, Witcomb GL, Nieder T, Nixon E, Yip A, Arcelus J. A comparison of mental health symptomatology and levels of social support in young treatment seeking transgender individuals who identify as binary and non-binary. Int J Transgend 2018; 20 (2-3): 241-50. http://dx.doi.org/10.1080/15532739.2018.1452660.

38. Rimes KA, Goodship N, Ussher G, Baker D, West E. Non-binary and binary transgender youth: Comparison of mental health, self-harm, suicidality, substance use and victimization experiences. Int J Transgend 2019; 20 (2-3): 230-40. http://dx.doi.org/10.1080/15532739.2017 .1370627

39. Vijlbrief A, Saharso S, Ghorashi H. Transcending the gender binary: Gender non-binary young adults in Amsterdam. J LGBT Youth 2019; 17 (1): 89-106. http:// dx.doi.org/10.1080/19361653.2019.1660295.

40. Zapata Pizarro A, Díaz Díaz K, Barra Ahumada L, Maureira Sales L, Linares Moreno J, Zapata Pizarro F. Atención de salud de personas transgéneros para médicos no especialistas en Chile. Rev Med Chile 2019; 147 (1): 65-72. https://dx.doi.org/10.4067/S003498872019000100065 . 